\title{
DESARROLLO DE LA COGNICIÓN SOCIAL EN PERSONAS CON TRASTORNO DE ESPECTRO AUTISTA
}

\author{
DEVELOPMENT OF SOCIAL COGNITION IN PEOPLE WITH AUTISM SPECTRUM \\ DISORDER
}

\section{Rosario Aguirre Martínez ${ }^{1}$}

\begin{abstract}
Resumen:
Introducción. El objetivo del artículo es realizar una revisión bibliográfica y responder a la pregunta ¿existen programas de intervención probados para desarrollar la cognición social en personas con trastornos del espectro autista (TEA)? Se define autismo según Kanner, Asperguer y Wing, este último que formuló la tríada sintomática del autismo. La Teoría de la mente (ToM), que es la capacidad para atribuir estados mentales a otras personas, distintos a los propios, da explicación a los 3 síntomas de Wing. Aportes desde la ToM. Se revisan distintos autores que aportaron en la investigación empírica de la ToM desde Premack y Woodruf (1978) que ocupan el concepto por primera vez, hasta Baron-Cohen (2001) que ha centrado sus investigaciones en estudios de la ToM en niños con autismo. Tratamientos propuestos para desarrollar la cognición social en personas con TEA. Se revisan 5 estudios que proponen diversas intervenciones para desarrollar la ToM en niños con TEA, el último realizado por Stichter y colaboradores el año 2012. Discusión. Se analiza la escasez de estudios con pequeñas poblaciones, lo que dificulta extrapolar los resultados. Se destaca la necesidad de realizar evaluaciones en contextos reales, profundizar los estudios de programas sistematizados que consideren grupo control, muestras representativas y seguimientos prolongados para evaluar la generalización de los aprendizajes. Finalmente se exponen aportes potenciales de la terapia ocupacional a los estudios y programas de tratamiento.
\end{abstract}

\section{Palabras claves:}

Trastorno espectro autista, teoría de la mente, cognición social.

\begin{abstract}
:
Introduction. The aim of this paper is to review the literature and answer the question: Do programs of intervention prove to develop social cognition in persons with disorders of the autistic spectrum? Autism is defined by Kanner, Asperguer and Wing, who formulated the symptomatic triad of autism. The Theory of the Mind (ToM), is the ability to attribute mental states to other persons, different from oneself, gives an explanation for three symptoms (Wing, Contributions of ToM). We review various authors who contributed by empirical research of ToM from Premack and Woodruff (1978) occupying the concept for the first time, to Baron-Cohen (2001) that centred his research on studies of ToM in children with autism. Treatments proposed to develop social cognition in persons with ASD. We reviewed 5 studies that propose diverse interventions to the development of ToM in children with ASD, the last one undertaken by Stichter and collaborators, in 2012. Discussion. We analyze the scarcity of studies with small populations, which makes it difficult to extrapolate results. The need for assessments in real contexts is analized; further studies of systematized programs of what is considered to be the control group; representative samples and follow-ups with a group prolonged to evaluate the generalization of the learning. Finally, we report potential contributions to occupational therapy research and treatment programs.
\end{abstract}

Key word:

Autism spectrum disorder, theory of mind, social cognition.

1 Terapeuta Ocupacional, licenciada en Modelo de Ocupación Humana, especialista en salud mental, postítulo en Integración Sensorial e Hipoterapia, candidata a Magíster en Desarrollo Cognitivo. E mail rosario_aguirrem@yahoo.es 


\section{INTRODUCCIÓN}

El autismo infantil fue descrito por primera vez en 1943 por Leo Kanner como una patología caracterizada por "falta de contacto con las personas, ensimismamiento y soledad emocional"; en 1944, Asperger describió el autismo como "un trastorno que produce dificultades considerables y muy típicas de integración social". Finalmente en 1981 Wing formuló la tríada sintomática del autismo que se refiere a deficiencias en: 1 . relaciones sociales, 2 . la comunicación y 3 . la capacidad de crear juegos de ficción (Portela, 2003).

La Teoría de la mente, abreviado ToM (theory of mind), que es la capacidad para atribuir estados mentales a otras personas, distintos a los propios, da explicación a los 3 síntomas descritos por Wing, sin embargo hay síntomas en el autismo que son independientes de las alteraciones sociales y pragmáticas como las reacciones al sonido, a la luz, al calor, y el tacto, que muestran algunas personas autistas, sin embargo en este artículo nos referiremos a la sintomatología propia de la ToM.

El objetivo de este artículo es realizar una revisión bibliográfica y responder a la pregunta ¿existen programas de intervención probados para desarrollar la cognición social en personas con trastornos del espectro autista (TEA)? Para dar respuesta a esta inquietud se buscaron artículos científicos que aborden el tema de Teoría de la Mente en personas con trastorno de espectro autista y tratamientos propuestos para los mismos.

\section{Aportes desde la Teoría de la Mente}

Premack y Woodruf (1978 citado en Tirapu-Ustárroz, Pérez, Erekatxo \& Pelegrín, 2007) ocupan por primera vez el concepto de ToM a raíz de estudios que realizaron con chimpancés, refiriéndose a la capacidad de atribuir estados mentales y predecir el comportamiento de otros. Realizaron un experimento con chim- pancés de laboratorio, a los que les mostraron un vídeo en el que se veía a uno de sus cuidadores encerrado en una jaula intentando alcanzar dos plátanos, uno que estaba colgando por encima del techo de la jaula y otro en el suelo, ambos fuera del alcance de la persona enjaulada. El humano tenía un piso para subirse y un palo manipulable a través de los barrotes. Cuando la persona iniciaba la acción para alcanzar los plátanos, los experimentadores fijaban la imagen y mostraban al chimpancé dos fotografías, una de ellas con la solución correcta que en el caso de los plátanos fuera de la jaula, mostraba la imagen del ser humano tomando un palo largo y sacándolo entre las rejas para alcanzar la fruta. La chimpancé Sarah acertó 21 veces de 24. Este estudio permitió concluir que los chimpancés poseen ToM, es decir son capaces de comprender las intenciones de las personas y anticipar sus acciones.

Posterior a estos estudios la ciencia cognitiva comienza los estudios sobre la ToM en seres humanos. Wimmer y Perner (1983, citado en Baron-Cohen, 1990), desarrollaron una prueba experimental sobre la comprensión del estado mental de "falsa creencia" por niños normales. La prueba se basa en una historia de muñecas que se le muestra a los niños, Sally y Anne (Figura 1), una vez explicada la historia se le pide al niño que señale dónde buscará Sally su pelota. Si el niño contesta que Sally buscará en su canasto, el niño puede entender que Sally tiene una creencia distinta a la suya, y por tanto falsa, entonces podemos decir que ha desarrollado ToM. Si el niño contesta que Sally buscará en la caja de Anne, el niño no puede entender que Sally no ha visto que Anne cambió la pelota a su caja, en este caso el niño no ha desarrollado ToM. Esta prueba no requiere lenguaje verbal, ya que se le pide al niño que señale el lugar y determina que la ToM se encuentra presente a los 4 años aproximadamente. Posterior a este estudio se realizan múltiples variaciones de la misma prueba para explorar los alcances de la ToM. 
Figura $\mathrm{N}^{\circ} 1$

Escenario de un test simple de teoría de la mente (Baron-Cohen, 1990)

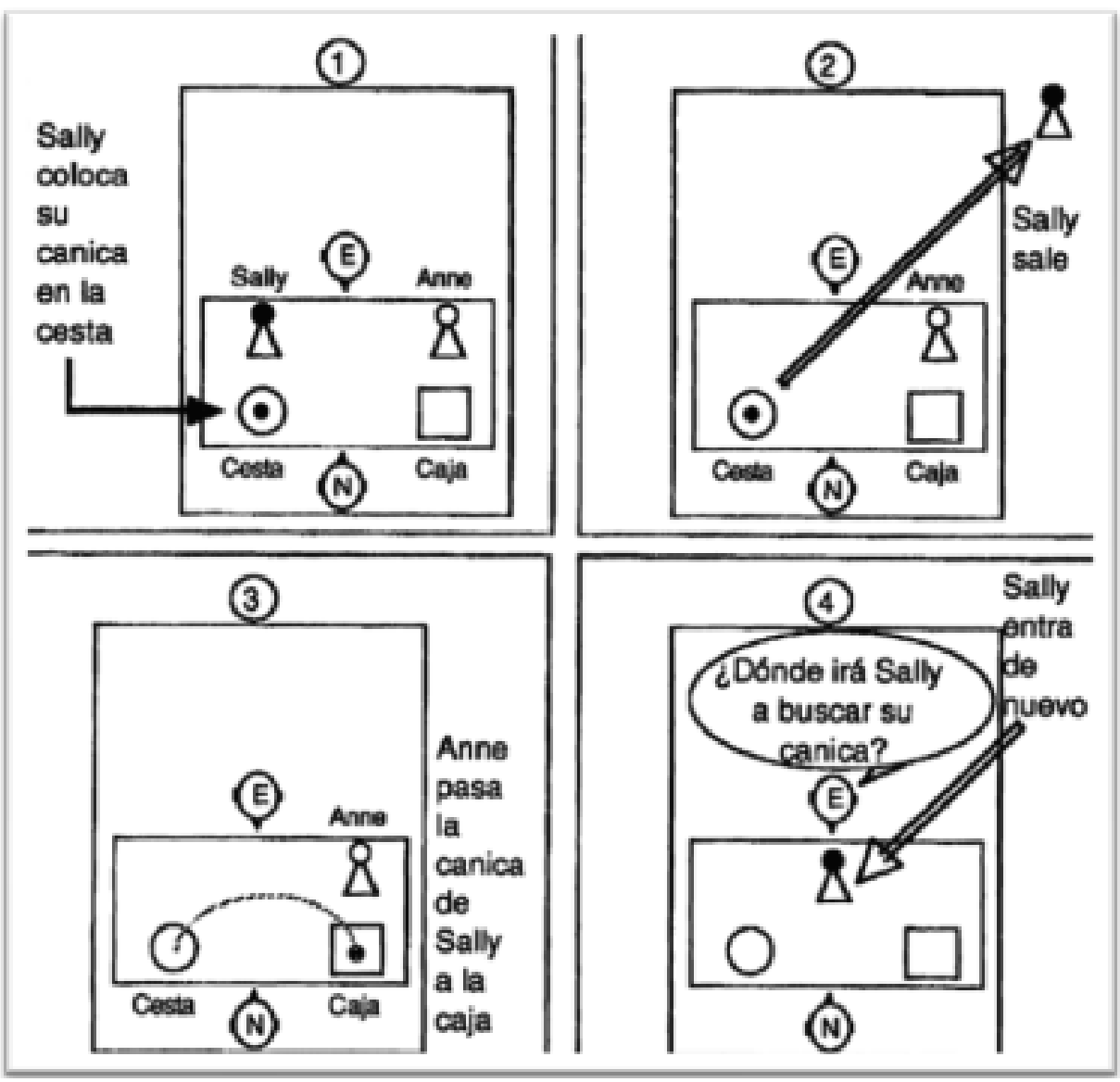

Baron-Cohen, Leslie y Fritz (1985) han centrado sus estudios en el autismo, son los primeros que desarrollan la prueba de Sally y Anne con niños normales, autistas y con síndrome de Down, encontrando que el $86 \%$ de los niños con síndrome de Down da la respuesta correcta (falsa creencia), y el $85 \%$ de los niños normales superaron la prueba, sólo el $20 \%$ de niños autistas acierta, a pesar de que este grupo tenía la misma edad mental y cronológica. Este resultado, proporciona una base preliminar a la hipótesis de que en el autismo hay un fallo en el desarrollo de una ToM, simultáneamente los datos de los grupos control revelan que tal déficit debe ser específico del autismo, y no el resultado de un retraso del desarrollo general, aplicando este criterio para el diagnóstico del los TEA.
A la prueba de falsa creencia de Sally y Anne, se la clasifica como "falsa creencia de primer orden", ya que pretenden medir la atribución a otros de creencias diferentes con las propias. Existen pruebas más complejas como "falsa creencia de segundo orden", que intentan medir la atribución a segundos de creencias de un tercero (Portela et al., 2003), como la prueba del heladero diseñada por Baron-Cohen (1989) donde se le relata a los niños la siguiente historia:

"Es un día caluroso de verano. Juan y María están sentados en el parque cuando ven llegar una furgoneta de helados. Como no llevan dinero encima, María decide ir a buscar la cartera a su casa. 
El heladero le asegura que esperará en el parque, pero al cabo de unos minutos Juan ve cómo el heladero arranca la furgoneta para irse. Al preguntarle dónde va, el heladero contesta que se marcha a la zona de la iglesia porque en el parque apenas hay gente. Cuando el heladero va conduciendo camino de la iglesia, María le ve desde la puerta de su casa y le pregunta dónde va. Así, María también se entera de que estará en la iglesia. Por su parte, Juan, que no sabe que María ha hablado con el heladero, va a buscarla a su casa pero no la encuentra. El marido de María le dice a Juan que ella se ha ido a comprar un helado" (Tirapu-Ustárroz et al., 2007 p 481).

Una vez relatada la historia se le pregunta al niño ¿Dónde piensa Juan que María habrá ido a buscar al heladero? Esta prueba resulta más compleja porque el niño tiene que hacer una atribución de lo que María piensa que Juan piensa. La atribución de creencia de segundo orden podrían resolverla niños normales de más de 7 años. Cuando el niño con autismo desarrolla la ToM, aunque a ritmo retrasado, puede seguir la misma secuencia del desarrollo normal (Baron-Cohen, 1990).

Happé (1995, citado en Tirapu-Ustárroz et al., 2007) crea la prueba de "historias extrañas" que son cuentos con ironía, mentiras y mentiras piadosas que buscan evaluar la habilidad de los niños autistas de atribuir intenciones a los demás. Un ejemplo de estas historias de mentiras piadosas es:
"Hoy tía Amelia ha venido a visitar a Pedro. Pedro quiere mucho a su tía pero hoy lleva un nuevo peinado que Pedro encuentra muy feo. Pedro cree que su tía está horrorosa con este pelo y que le quedaba mucho mejor el que llevaba antes. Pero cuando tía Amelia le pregunta a Pedro: '¿Qué te parece mi nuevo peinado?', Pedro dice 'iOh, estás muy guapa!. Pregunta: ¿por qué le dice eso Pedro?" (Tirapu-Ustárroz et al., p. 482).

Con esta prueba Happé plantea la necesidad de coherencia central o global, donde el niño debe superar la literalidad de la historia para generar un significado en un contexto concreto.

Howlin (2008) cita en su artículo, un estudio no publicado Read (1996) donde se prueba que las personas con TEA dan más importancia a las claves físicas que a las claves sociales. En la prueba presentó a tres grupos de adultos jóvenes (TEA, esquizofrenia y desarrollo típico, igualados en edad y cociente intelectual) una serie de historias ilustradas que implicaban situaciones sociales difíciles o problemáticas (fig. 2). Se les pidió a los participantes que completaran cada historieta con uno de los dos dibujos que se les daba como alternativas. Las personas con TEA, tenían en cuenta la clave física (p. ej., la vela es más grande en el dibujo final o la bañera desbordándose) y respondían menos en función del contexto social de la historieta. Los otros participantes con desarrollo típico o esquizofrenia, tendían a no notar la clave física anómala, sino que respondieron a las claves sociales. 
Figura $\mathrm{N}^{\mathrm{o}} 2$

Relevancia de las claves visuales frente a las sociales (Howlin, 2008)

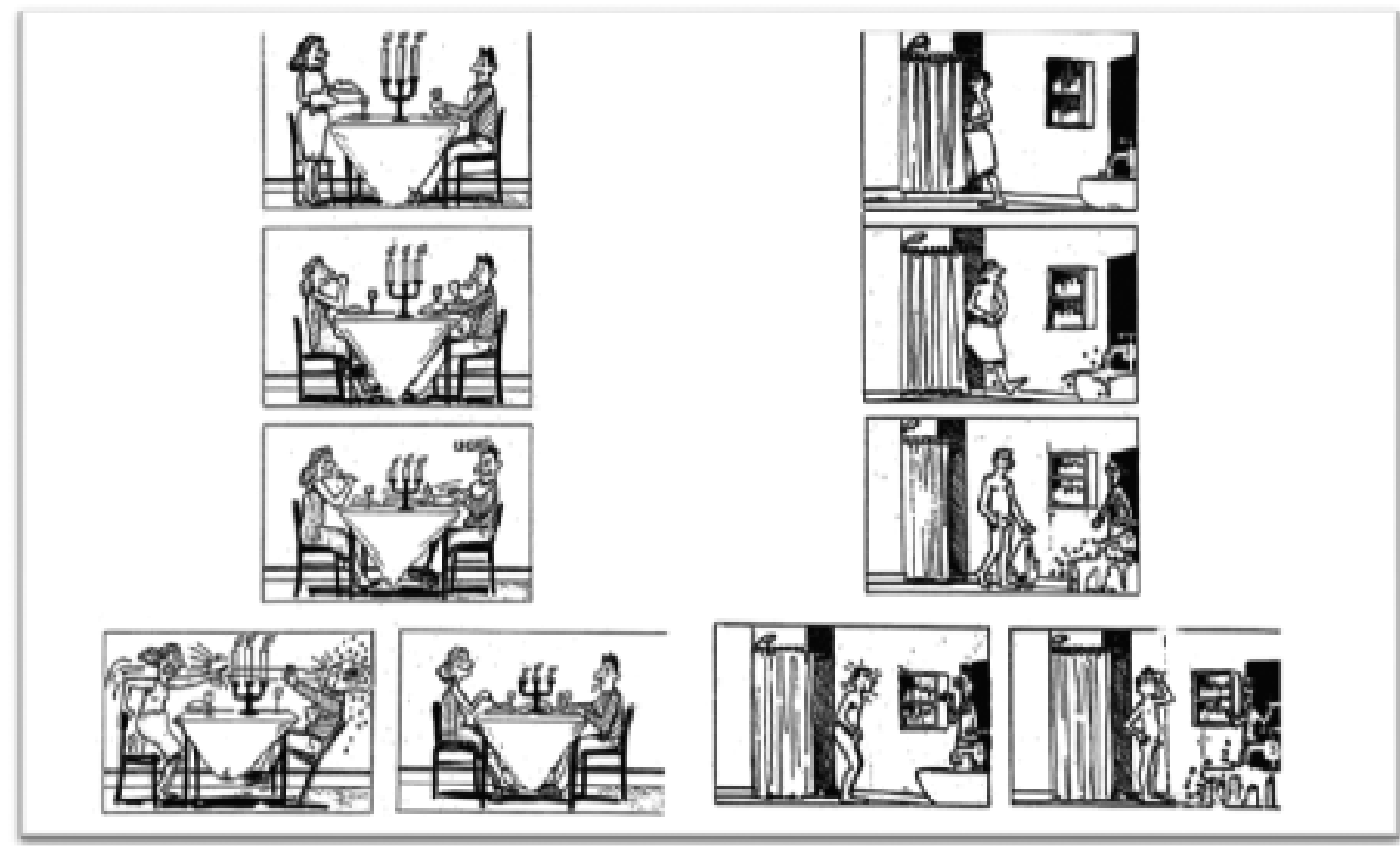

El grupo de Baron-Cohen (1999) propuso un nuevo test para la valoración de la sensibilidad social, que permitía diferenciar la ejecución de niños normales de la ejecución de niños de 7 a 11 años, afectados por el síndrome de Asperger. En esta prueba, los sujetos deben leer 10 historias en las que el protagonista "mete la pata" en distintas situaciones sociales y 10 historias de control de tipo "aséptico". Una de las historias de faux pas es la siguiente:

"Julia compró a su amiga Esther un jarrón de cristal como regalo de bodas. Esther hizo una gran boda y había tal cantidad de regalos que le fue imposible llevar la cuenta de qué le había regalado cada invitado. Un año después, Julia estaba cenando en casa de Esther. A Julia se le cayó una botella de vino sin querer sobre el jarrón de cristal y éste se hizo añicos. 'Lo siento mucho. He roto el jarrón' dijo Julia. 'No te preocupes, dijo Esther, nunca me gustó; alguien me lo regaló por mi boda." (Tirapu-Ustárroz et al., 2007 p. 482).
Se le pregunta al niño: ¿ Ha dicho alguien algo que no debería haber dicho o algo inoportuno? Esta prueba evalúa la capacidad de comprender el significado no literal de las aseveraciones y ponerse en el lugar de los diferentes protagonistas (empatía). En este estudio se muestra que las puntuaciones de faux pas eran significativamente más altas en los niños de 11 años y que las calificaciones fueron mayores para las mujeres que los varones.

Baron-Cohen (2001, citado en Tirapu-Ustárroz et al., 2007) avanza en sus estudios y el año 2001 crea el test de la mirada, que valora aspectos más complejos que son el reconocimiento de las emociones y sentimientos. Esta prueba consta de 28 fotografías para niños y 38 para adultos en las que se observan las miradas de hombres y mujeres que expresan un sentimiento o pensamiento. Cada fotografía tiene cuatro respuestas posibles que aparecen en la pantalla y el sujeto debe elegir la más adecuada. En este estudio se constata que los adultos con autismo o síndrome de Asperguer puntuaban bajo en el test de la mirada (Valdez, 2001).

Un precursor de la ToM sería la habilidad para comprender estados atencionales de las personas, como demuestra Bruner (1983, citado en Baron-Cohen, 1990) 
en la prueba "atención conjunta" en niños pequeños, estas conductas implican la coordinación visual propia con la de otro, incluyen dar y mostrar objetos, y señalar objetos. Señalar se produce cuando una persona extiende su dedo índice hacia un objeto, esto involucra dos funciones al menos: la primera es para obtener un objeto, llamada "señalar protoimperativo" ${ }^{2}$, mientras que la segunda es para llamar la atención con la intención de comentar o mostrar interés por un objeto, Ilamada "señalar protodeclarativo". Esta conducta se desarrolla muy tempranamente, y está presente alrededor de los 10-14 meses de edad.

Charman y cols. (2000, citado en Howlin, 2008) detalla el desarrollo de la atención conjunta, que surge de seguir la mirada de otro, alrededor de los 6 meses; compartir la mirada, 9 a 12 meses y de la indicación protodeclarativa, señalar para dirigir la atención de otros a objetos de interés, más o menos a los 12 meses de edad.

Los niños autistas son muy pobres en cuanto a la cantidad de conductas de atención conjunta, mientras que usan y comprenden el señalar protoimperativo, casi nunca utilizan o comprenden el señalar protodeclarativo (Baron-Cohen, 1990). Esto sugiere que el diagnóstico temprano del autismo podría ser posible en base a la ausencia de conductas de atención conjunta.

Los distintos estudios revisados anteriormente nos han demostrado que la ToM nos permite:

- Sintonizar con la mirada de otro.

- Entender estados mentales y predecir el comportamiento de otras personas.

- Superar la literalidad de comportamientos sociales para comprender ironías, bromas, mentiras y mentiras piadosas.

- Pasar por alto detalles del ambiente físico para comprender claves sociales.

- Reconocer emociones y sentimientos de otras personas en su mirada.

- Empatizar.

2 Proto, prefijo procedente del griego "prótos" que significa primero. Los conceptos "protoimperativo" y "protodeclarativo" han sido utilizados por la comunidad científica para referirse a actos comunicativos intencionales en el periodo preverbal del desarrollo.
Todas estas conductas son fundamentales en la vida de las personas, son necesarias desde encontrar la mirada de otra persona para pedir un objeto o señalar interés, por ejemplo cuando se va a comprar, participar en instancias grupales como la educación, entender las emociones de los otros para establecer vínculos con familiares, etc. Las dificultades en la cognición social afectan todas las áreas de desempeño de las personas.

Frente a este escenario surge la pregunta: ¿se puede desarrollar la cognición social en personas con TEA?, ¿Qué tipo de intervención es más efectiva? Para dar respuesta a estas preguntas, se realizó una revisión bibliográfica de estudios que han documentado intervenciones terapéuticas con base empírica, en los últimos 20 años.

\section{Tratamientos propuestos para desarrollar la cognición social en personas con TEA}

Frith (1994, citado en Miguel, 2006) plantea que la actuación en tareas que impliquen la comprensión de emociones, creencias e intenciones de los demás, se encuentra estrechamente relacionada con el nivel de competencia social, por lo cual los niños que resuelvan con éxito estas tareas, lograrán mayores niveles de adaptación social, por ello la labor de los estudiosos es acercarnos al mundo autista para que los sujetos con TEA, puedan comprender las emociones y facilitarles el proceso comunicativo, utilizando todos los medios disponibles y que permite la tecnología existente.

Son múltiples las investigaciones que abordan las deficiencias de la ToM en las personas con TEA (véase Portela et al., 2003; López \& Rejón, 2005; Lecannelier, 2004 y Gómez, 2010), sin embargo son escasos los estudios que profundizan en abordajes terapéuticos efectivos a partir de estos modelos explicativos, es por este motivo que se ha querido sistematizar estas experiencias, que aunque son escasas, resultan orientadoras en la intervención. A continuación se detallan estudios empíricos que han detallado programas para desarroIlar la ToM en personas con TEA.

Fisher y Happé (2005) realizaron un estudio de entrenamiento con niños diagnosticados con TEA, 10 niños fueron capacitados en la teoría de la mente, 10 fueron entrenados en la función ejecutiva y 7 niños fueron asignados a un grupo de control, que no recibió la intervención. Los programas de entrenamiento se administraron de forma individual, durante 5 y 10 días con una duración de 25 minutos. Los niños fueron evaluados antes del entrenamiento, después del entrena- 
miento en un período de dos meses de seguimiento. Se observaron mejoras significativas en el rendimiento en tareas de teoría de la mente en el $50 \%$ de los niños de ambos grupos, mientras que el grupo control no mostró mejoría, y tampoco se observó mejoría de la función ejecutiva en ningún grupo.

Chin y Opitz (2000) realizan un estudios con 3 niños con autismo de alto funcionamiento, donde se les enseña cómo iniciar una conversación, turnarse durante la conversación, escuchar con atención, mantener un tema de conversación, y cambiar el tema de conversación apropiadamente. Los niños fueron evaluados para ToM utilizando tareas de falsa creencia antes y después de las sesiones de entrenamiento. Las sesiones se Ilevaron a cabo dos veces por semana durante 1 hora, no se especifica el número de sesiones totales. Los resultados indican que la cantidad de interés común mostrada por los niños con autismo durante la conversación con sus cuidadores se incrementó durante las sesiones de entrenamiento. Los niños también dieron un mayor número de respuestas apropiadas para el contexto de la conversación, sin embargo el rendimiento en las tareas de falsa creencia se mantuvo constante durante todo el estudio. Ozonoff y Miller (1995) realizaron un estudio parecido con 5 niños y obtuvieron similares resultados.

Lacava, Golan, Baron-Cohen y Smith-Myles (2007) realizaron un estudio donde utilizaron el DVD "Lectura Mental: Una Guía Interactiva sobre las Emociones" para enseñar el reconocimiento de emociones a 8 niños con TEA de edades comprendidas entre los 8 y 11 años. La intervención consistió en 10 semanas de uso del programa informático, en el hogar o el entorno escolar. Se evaluó pre y post intervención, los resultados indicaron que después de la intervención, los participantes mejoraron su capacidad para reconocer emociones complejas en la cara y la voz.

Lozano y Alcaraz (2010) realizan un estudio de 3 casos, dos alumnos de educación primaria y una alumna de educación secundaria con TEA, donde se diseñaron y elaboraron materiales didácticos dirigidos a la enseñanza de la comprensión de emociones y creencias, y un protocolo de entrevista de valoración de habilidades sociales para personas con TEA. El entrenamiento se realizó 2 veces por semana en sesiones de 45 minutos, por 11 semanas, con un total de 22 sesiones; la metodología consistió en la utilización de recurso didáctico "Aprende con Zapo. Propuestas didácticas para el aprendizaje de habilidades emocionales y sociales" creado por la misma autora, la enseñanza se realizó en un entorno multimedia-interactivo como apoyo al material impreso y seminarios de acción y reflexión. Los alumnos fueron evaluados antes y después del proceso de intervención educativa sobre sus niveles de comprensión de emociones y creencias y habilidades sociales. Se recurrió a la estadística descriptiva para el procedimiento de análisis de datos cuantitativos, y al análisis de contenido de las observaciones, entrevistas y comentarios de maestros y padres para el análisis de datos cualitativos. Los resultados indican que, tras el proceso de intervención educativa, los alumnos participantes en la investigación mejoraron su rendimiento en la comprensión de emociones y creencias.

Stichter et al. (2012) describen el desarrollo y la implementación de una intervención de grupo basada en competencia social, enfocándose en función ejecutiva y ToM. Participaron 20 estudiantes con edades entre 11 y 14 años con diagnóstico de TEA de alto funcionamiento; el tratamiento se llevó a cabo durante 10 semanas, 2 veces por semana, con un total de 20 horas. Al final del estudio los padres y los profesores reportaron mejorías significativas en las habilidades sociales y función ejecutiva de los hijos (evaluada con test específicos).

Hasta ahora se han revisado estudios que se refieren a la dificultad de los niños con TEA para manejar la ToM y trabajos en los que se proponen programas para enseñar habilidades de cognición social. Browler y Stromm (1998 citado en Howlin, 2008) refieren que la mayoría de los estudios en este campo se concentran en la enseñanza de la prueba de falsa creencia, mejorando el rendimiento en estas pruebas, sin embargo no se produce generalización a otras conductas requeridas cotidianamente. Para los mismos fines se utilizaron los globos de pensamiento que se ocupan comúnmente en libros de niños, sin embargo Wellman y colaboradores (2002 citado en Howlin) aseveran que no en todos los niños con TEA son útiles estas estrategias.

3 Aprende con Zapo, es un recurso didáctico creado por Josefina Lozano y Salvador Alcaraz, autores del artículo citado, que ha sido comercializado por la editoral Wolters Kluwer España Educación. 


\section{DISCUSIÓN}

En esta revisión se ha presentado información actualizada referente a las implicaciones del déficit de ToM en las personas con TEA, intentado dar respuesta a la pregunta ¿existen programas de intervención probados para desarrollar la cognición social en personas con trastornos del espectro autista (TEA)?

En los estudios experimentales revisados se ha observado resultados positivos en poblaciones pequeñas, de 3 a 20 personas, con intervenciones de una vez por semana durante 10 días como mínimo, sin embargo las técnicas de intervención propuestas y el alcance de las mismas han sido poco exploradas, lo que no permite extrapolar los resultados en otras poblaciones.

Por otro lado, la mayoría de los autores advierten la necesidad de métodos adicionales para mejorar la generalización de los aprendizajes, ya que los seguimientos más prolongados son de 2 meses. Rogers (2000 citado en Lozano \& Alcaraz, 2010) refiere que las dificultades de generalización de los aprendizajes en los niños y jóvenes con TEA, es posible que respondan a la falta de enseñanza sistemática y explícita en contextos naturales.

Se requiere profundizar los estudios de programas sistematizados que consideren grupo control, muestras representativas y seguimientos prolongados para evaluar la generalización de los aprendizajes en la vida cotidiana.

Sería conveniente desarrollar evaluaciones de la cognición social, que cuantifiquen estas habilidades en contextos cotidianos, ya que las estimaciones en ambientes experimentales añaden un grado de dificultad a la expresión de la conducta social. Se requiere mayor coherencia entre las evaluaciones y los tratamientos propuestos para desarrollar la ToM.

En lo referente a la intervención grupal para desarrollar habilidades de cognición social en personas con TEA, surge la inquietud si esta será la modalidad más adecuada como primera aproximación terapéutica en personas que precisamente tienen "ceguera de la mente" (Baron-Cohen, 1990) para leer las emociones y las intenciones de otras personas.

Con los antecedentes estudiados, y mi experiencia profesional, se plantea desarrollar habilidades de cognición social en niños con TEA, con programas sistemáticos, que deben realizarse en 2 momentos: primero una intervención individual donde se entreguen herramientas a las personas con TEA para comprender el lenguaje de las emociones y/o claves sociales utilizando material de apoyo visual y posteriormente intervencio- nes grupales o prácticas en la vida cotidiana, donde se ponga en práctica la interacción con otros utilizando estrategias de acción y reflexión.

La terapia ocupacional es una disciplina que se ha dedicado a estudiar el desempeño de las personas en las actividades de la vida cotidiana (autocuidado, ocio, juego, trabajo y estudio) y a la intervención en cada una de estas áreas para mejorar su calidad de vida. Dada esta versatilidad de ambientes en que se sitúa el terapeuta ocupacional es posible aportar en programas de evaluación y desarrollo de la cognición social en la vida diaria de las personas con TEA, con estrategias de acción y reflexión propias de la profesión.

El Ministerio de Salud de Chile, en la Guía Clínica de detección y diagnóstico oportuno de los TEA (2011), recomienda la participación de los terapeutas ocupacionales en el diagnóstico, tratamiento y rehabilitación de las personas con TEA, sin embargo existen escasas publicaciones que documenten la experiencia de los colegas en esta área, en la revisión bibliográfica en Lilacs y Ebsco, se encontraron solo 2 artículos que abordan esta problemática: "La alimentación en los trastornos del espectro autista desde la terapia ocupacional" (Cáceres, 2012) y "Déficit de procesamiento sensorial en el espectro del autismo" (Imperatore \& Reinoso, 2007).

Conociendo programas de terapia ocupacional dedicados a tratar y rehabilitar personas con TEA en varios hospitales para niños de Santiago, no queda duda que hace falta documentar dichos programas.

\section{AGRADECIMIENTOS}

A Sonia Oyarzún, psicóloga y amiga que me introdujo en el tema de la cognición social, y Natalia Salas por la motivación a escribir este artículo.

\section{ReferenCias Bibliográficas}

Baron-Cohen S. (1990) Autismo: un trastorno cognitivo específico de "ceguera de la mente". International Review of Psychiatry, 2, 81-90.

Baron-Cohen, Leslie, A. \& Frith, U. (1985) Does the autistic child have a "theory of mind"? Cognition, 21, 37-46.

Baron-Cohe S.; O'Riordan, M.; Stone, V.; Jones, R. \& Plaisted, K. (1999) Recognition of Faux Pas by Normally Developing Chil- 
dren and Children with Asperger Syndrome or High-Functioning Autism. Journal of Autism and Developmental Disorder, 29 (5), 407-417.

Cáceres, I. (2012). La Alimentación en los Trastornos del Espectro Autista desde la Terapia Ocupacional. Rev Retocyl 3, 11-13.

Chin, H. \& Opitz, B. (2000). Teaching conversational skills to children with autism: effect on the development of a theory of mind. Journal of Autism and Developmental Disorders. 30 (6), 569-583.

Gómez, I. (2010). Ciencia Cognitiva, teoría de la mente y autismo. Pensamiento Psicológico, 8 (15), 113-123.

Howlin, P. (2008). ¿Se puede ayudar a los niños con trastornos del espectro autista a adquirir una teoría de la mente? Revista de Logopedia, Foniatría y Audiología, 28 (2), 74-81.

Imperatore, E. \& Reinoso, G. (2007). Revisión de la Literatura: Déficit de procesamiento sensorial en el espectro del autismo. Rechto, $\mathrm{N}^{\circ} 7,59-68$.

Lacava, P.; Golan, O.; Baron-Cohen \& Smith, B. (2007). Using Assistive Technology to Teach Emotion Recognition to Students With Asperger Syndrome. A Pilot Study. Remedial and Special Education. 28 (3), 174-181.

Lecannelier, F. (2004). Los aportes de la teoría de la mente a la psicopatología del desarrollo. Terapia psicológica, 22 (1), 61-67.

López, JM. \& Rejón, C. (2005). Origen y destino de la teoría de la mente: su afección en trastornos distintos del espectro autista. Psiquiatría Biológica, 12 (5), 42-54.

Lozano, J. \& Alcaraz, S. (2010). Enseñar emociones para beneficiar las habilidades sociales de alumnado con trastornos del espectro autista. Educación Siglo XXI, 28, 261-268.

Miguel, A. (2006). El mundo de las emociones en los autistas. Revista Electrónica Teoría de la educación y Cultura en la Sociedad de la Información, 7(2), 169-180. Extraído el 13 de mayo, 2013 de http://campus.usal.es/ teoriaeducacion/rev_numero_07_02/n7_02_ana_miguel.pdf

Chile, Ministerio de Salud (2011). Guía de práctica clínica. Detección y diagnóstico oportuno de los trastornos del espectro autista (TEA).

Portela, V.; Vírseda, A. \& Gayubo, L. (2003). Revisión sobre el estudio de la teoría de la mente y trastornos generalizados del desarrollo y esquizofrenia. Actas Psiquiatría, 31 (6), 339-346.

Stichter P. et al. (2012). Social Competence Intervention for Elementary Students with Aspergers Syndrome and High Functioning Autism. J Autism Dev Disord, 42, 354-366.

Tirapu-Ustárroz, Pérez, G.; Erekatxo, M. \& Pelegrín, C. (2007). ¿Qué es teoría de la mente? Revista de neurología, 44 (8), 479-489.

Valdez, D. (2001). Teoría de la Mente y espectro autista. En Autismo: enfoques actuales para padres y profesionales de la salud y la educación. Extraído el 13 de mayo, 2013 de Espectroautista.info: http://campus.usal.es/ teoriaeducacion/rev_numero_07_02/n7_02_ana_miguel.pdf 